\title{
STOMACH
}

\section{Trefoil factor 1 is required for the commitment programme of mouse oxyntic epithelial progenitors}

\author{
S M Karam, C Tomasetto, M-C Rio
}

Gut 2004;53:1408-1415. doi: 10.1136/gut.2003.031963

\begin{tabular}{|c|}
\hline \\
\hline $\begin{array}{l}\text { Correspondence to: } \\
\text { Dr S M Karam, } \\
\text { Department of Anatomy, } \\
\text { Faculty of Medicine and } \\
\text { Health Sciences, UAE } \\
\text { University, Al-Ain, PO Bo } \\
\text { 17666, United Arab } \\
\text { Emirates; } \\
\text { skaram@uaeu.ac.ae }\end{array}$ \\
\hline $\begin{array}{l}\text { Revised version received } \\
4 \text { February } 2004 \\
\text { Accepted for publication } \\
17 \text { March } 2004\end{array}$ \\
\hline
\end{tabular}

See end of article for authors' affiliations

Correspondence to: Department of Anatomy Faculty of Medicine and University, Al-Ain, PO Box 17666, United Arab Emirates;

4 February 2004

17 March 2004
Background: Trefoil factor 1 (TFF1/pS2) is a major secretory product of the stomach and TFF1 knockout mice constantly develop adenomas and occasional carcinomas in the pyloric antrum.

Aim: To analyse the role of TFF1 in the differentiation of gastric epithelial cell lineages using oxyntic mucosae from normal and TFF1 knockout mice.

Methods: The various cell lineages were labelled using specific markers of pit, neck, parietal, and enteroendocrine cells. Patterns of TFF1, TFF2, and TFF3 expressions were defined using western blotting, immunohistochemistry, and/or immunogold electron microscopy.

Results: In normal mice, starting from postnatal day 1 (P1), TFF1 and TFF2 were produced by mucus secreting cells of the developing epithelium. At P7, TFF3 expression occurred in pit and parietal cells. When oxyntic glands were compartmentalised, at P21 and in older mice, TFF1 and TFF2 were expressed in pit and neck cells, respectively, and TFF3 was no longer in parietal cells but became a feature of zymogenic cells. In TFF1 deficient mice, alteration of oxyntic epithelial differentiation became obvious at P21, showing significant amplification of pit cells at the expense of parietal cells. At the molecular level, lack of TFF1 induced dramatic inhibition of TFF2 expression and more precocious TFF3 expression.

Conclusion: In the oxyntic mucosa, all three TFFs are produced in a lineage specific manner and TFF1 is essential in maintaining the normal commitment programme of epithelial progenitors.
$\mathrm{T}$ he mouse stomach includes four main regions: fundus, cardia, oxyntic region (corpus or body), and pyloric antrum. The fundus is lined by stratified squamous epithelium whereas a simple columnar epithelium lines the three other regions, forming numerous pits continuous with mucosal glands. ${ }^{1}$ In the oxyntic region, the pits are short and contain mucus secreting pit cells and some acid secreting parietal cells whereas the glands are long and contain mucus secreting neck cells, pepsinogen/intrinsic factor producing zymogenic cells, and parietal cells (fig lA). Enteroendocrine cells are also scattered in the pit-gland units. In addition, epithelial progenitors anchored in the isthmus, a small glandular region located next to the pit boundary (fig lA), are responsible for continuous renewal of all mature cells. ${ }^{2}$ It is generally believed that alteration of the progenitor cells is an early event that occurs during cancer development. Proliferation and differentiation of these cells also represents a crucial event that occurs during mucosal injury and healing. Therefore, it is important to define factors that control the normal proliferation/commitment programme of these epithelial progenitors.

Trefoil factors (TFFs) may play a role in the control of these progenitor cells. ${ }^{4}$ TFFl was discovered during differential screening of a cDNA library from a human breast cancer cell line, MCF-7. ${ }^{5}$ TFFl is normally expressed by gastric epithelial cells and is found in humans as a component of gastric juice. TFF 1 plays a protective role against mucosal injury ${ }^{7}$ and development of antropyloric adenoma. ${ }^{8}$ The latter is due to delaying cell progression from Gl to $S$ phase and downregulation of apoptosis.'

TFF2 or spasmolytic polypeptide was identified in porcine pancreatic secretion and neck cells of mouse and human stomachs. ${ }^{10-12}$ Due to its effect on acid secretion and cell proliferation, TFF2 has a protective role against gastric ulceration. ${ }^{13}$

While TFF 1 and TFF 2 are predominantly produced in the stomach, their roles may extend to the small and large intestines in cases of ulcer and Crohn's disease. ${ }^{14}$ Their ectopic expression was restricted to the regenerative ulcer associated cell lineage. ${ }^{15}$

TFF3 was identified in goblet cells of rat small and large intestines but not in normal stomach. ${ }^{16}$ Recently, it has been shown that TFF3 is synthesised by gastric cancer cells while expression of TFF 1 and TFF2 is downregulated. ${ }^{17}$

The aim of this study was to examine the oxyntic mucosa of normal and TFFl deficient mice to characterise: (1) immunolocalisation of TFFs and determine whether they are expressed in epithelial progenitors and (2) effects of TFFl deficiency on expression of other TFFs and the commitment programme of epithelial progenitors.

\section{MATERIAL AND METHODS}

Animals

C57BL/6J/129/Svj mixed genetic background mice of both sexes were studied on postnatal days 1 (P1), P3, P7, P14, P21, $\mathrm{P} 30$, and $\mathrm{P} 60$ ( $\mathrm{n}=3-7$ for each age group). Similar age groups and numbers of TFFl knockout mice ${ }^{8}$ were maintained in a pathogen free state and given a standard chow diet ad libitum. To label cells in $\mathrm{S}$ phase, some mice received an intraperitoneal injection of 5-bromo-2'-deoxyuridine (BrdU $120 \mathrm{mg} / \mathrm{kg}$ ) one hour before sacrifice.

\section{Generation of antibodies}

Synthetic peptides corresponding to the carboxy terminal ends of TFFs (TFF1, FHPMAIENTQEEECPF; TFF2, EPVWCFFPQSVEDCHY; TFF3, IPGVPWCFKPLQEAECTF) were coupled to ovalbumin and injected into New Zealand rabbits. Immunoreactive sera were affinity purified against the synthetic peptides linked to sulfolink coupling gel (Pierce, Rockford, Illinois, USA). Recombinant mouse TFF1, TFF2,

Abbreviations: TFF, trefoil factor; EM, electron microscopy; BrdU, 5bromo-2"-deoxyuridine; P, postnatal day; UEA, Ulex europaeus agglutinin; GSII, Grifforia simplifolica II 

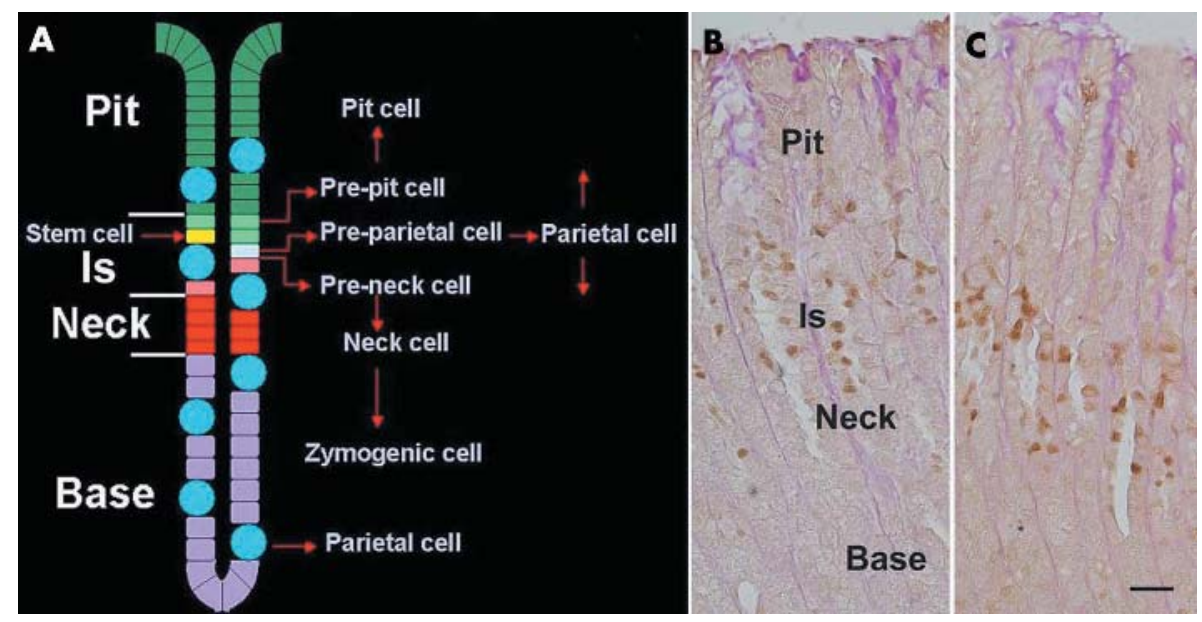

Figure 1 (A) Diagram of epithelial organisation in the mouse oxyntic mucosa. Cells form a short pit opening into a gland composed of isthmus (Is), neck, and base. In the isthmus, the mutipotent stem cell gives rise to committed progenitors (pre-pit, pre-parietal, and pre-neck cells) which are responsible for the production of the four main cell types: pit, parietal, neck, and zymogenic cells. (B) Normal mouse oxyntic mucosa at postnatal day (P) 60, showing proliferating cell nuclear antigen (PCNA) labelled cells with brown nuclei which are mainly located in the isthmus (Is). (C) PCNA labelling in trefoil factor 1 knockout mouse (P60) showing a normal pattern of cell proliferation. Bar $=30 \mu \mathrm{m}$.

and TFF3 proteins were obtained using CHO cells transfected with expression vectors using jetPEI transfection reagent (Polyplus Transfection, Illkirch, France). After 24 hours, cells were washed twice in serum free medium and incubated for 24 hours in chemically defined medium (Gibco CD CHO medium; Invitrogen, Grand Island, New York, USA). Conditioned media were collected and dead cells removed by centrifugation. Protein pellets were acetone precipitated, air dried, and suspended in loading buffer. Proteins were resolved by tricine sodium dodecyl sulphate-polyacrylamide gel electrophoresis ${ }^{18}$ and electrotransferred to nitrocellulose sheets (Schleicher and Schuell, Dassel, Germany). Membranes were probed with anti-TFF1, anti-TFF2, and anti-TFF3 antibodies at dilutions of 1/2000, 1/20 000, and $1 / 2000$, respectively. After washing, blots were incubated with horseradish peroxidase conjugated AffiniPure donkey antirabbit immunoglobuling (IgG) at 1/10 000 (Jackson ImmunoResearch, West Grove, Pennsylvania, USA). Protein-antibody complexes were visualised by an enhanced chemiluminescence detection system (SuperSignal, West Pico; Pierce) (fig 2A).

Light microscopic and immunohistochemical analysis Pieces of stomach cut along their longitudinal axis were fixed in Bouin's solution and embedded in paraffin. Some sections $(5 \mu \mathrm{m})$ were stained with haematoxylin-eosin or periodic acid Schiff for general histology. Other sections were used for immunohistochemistry or lectin binding. Sections were examined with a fluorescence microscope.

The lineage specific primary antibodies used were rabbit anti-HKalphaN $2^{19}$ specific for the alpha subunit of the $\mathrm{H}, \mathrm{K}$ ATPase of parietal cells. Polyclonal anti-intrinsic factor ${ }^{20}$ and anti-ghrelin $^{21}$ antibodies were utilised as markers for zymogenic and enteroendocrine cells, respectively. Mitotic cells were labelled using goat anti-BrdU ${ }^{22}$ and antiproliferating cell nuclear antigen antibodies. Tetramethylrhodamine isothiocyanate labelled Ulex europaeus type 1 agglutinin (UEA-1) and fluorescine isothiocyanate labelled Grifforia simplifolica II
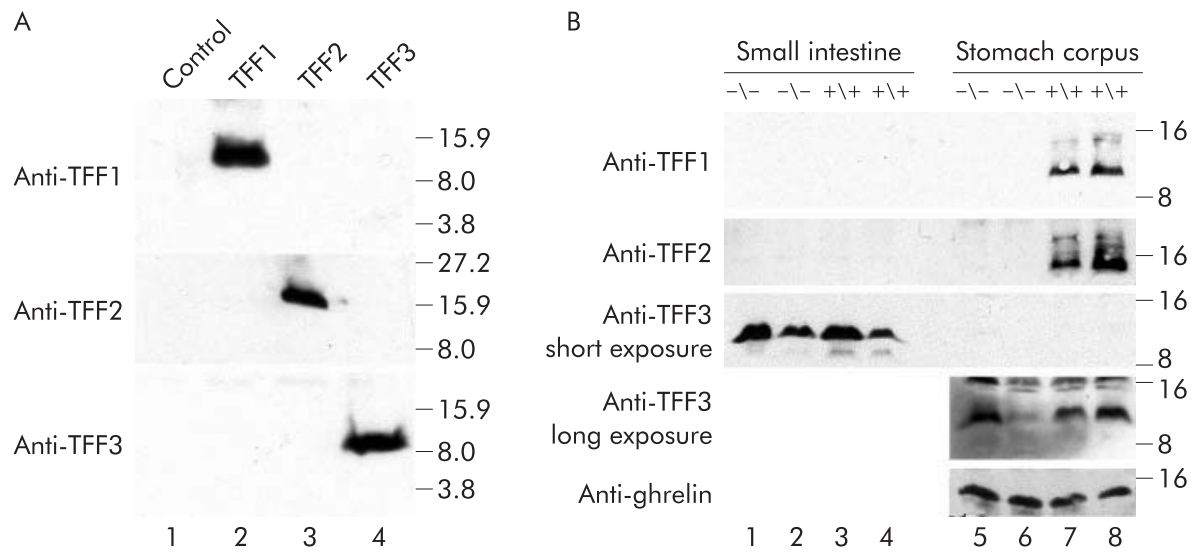

Figure 2 (A) Specificity of anti-trefoil factor (TFF) antibodies. Western blots containing $150 \mu \mathrm{l}$ of acetone precipitated proteins from $\mathrm{CHO}$ cells transfected with empty vector (lane 1) and vectors expressing TFF1 (lane 2), TFF2 (lane 3), and TFF3 (lane 4) were probed with anti-TFF antibodies. No cross reaction was observed. (b) Expression of TFFs in the small intestine (lanes 1-4) and stomach corpus (lanes 5-8) of two normal (+/+) and two TFF 1 deficient (-/-)mice. For anti-TFF1 and anti-TFF2 probing, each lane contained $20 \mu \mathrm{g}$ of total protein extracts (exposure time five seconds); for antiTFF3, each lane contained acetone precipitated proteins from $400 \mu \mathrm{g}$ of total protein extracts (exposure times five seconds and 30 minutes). Antighrelin antibody was used as the loading control. ${ }^{21}$ TFF1 and TFF2 proteins were only present in the stomach corpus of normal mice. Irrespective of TFF1 status, high and low TFF3 levels were observed in the intestine and stomach corpus, respectively. Antibodies used (left) and protein size markers (right) are indicated. 

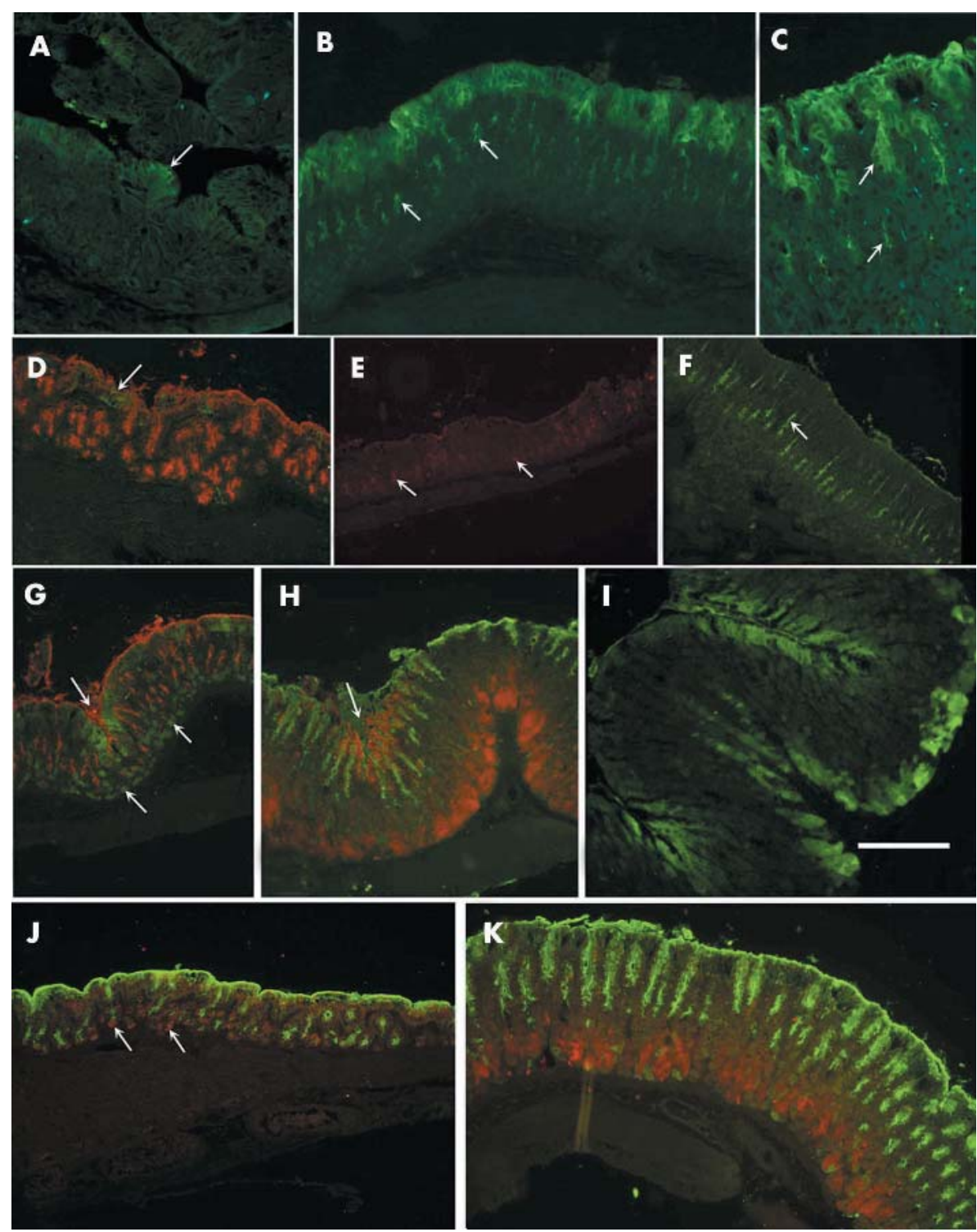

Figure 3 Immunohistological localisation of trefoil factors (TFFs) during postnatal development of the oxyntic mucosa of normal (A-I) and TFF1 knockout $(J, K)$ mice. Paraffin sections were incubated with rabbit antimouse TFF1 (A-C), TFF2 (D-F), and TFF3 (G-K). Antigen-antibody binding sites were visualised by the fluorescine isothiocyanate (FITC) conjugated goat antirabbit lgG (green: A-D, F, G, I) or with tetramethylrhodamine isothiocyanate (TRITC) conjugated goat antirabbit lgG (red: E, H, J, K). Mucus secreting pit cells were labelled with either TRITC (red: D, G) or FITC (green: H, J, K) conjugated to lectin. (A) P1: folded epithelial lining exhibiting small patches of TFF1 expressing cells (arrow). (B) P3: pit cells lining the luminal surface labelled with anti-TFF1 as some cells in the developing epithelial buds (arrows). (C) P21: localisation of TFF1 in the cells lining the luminal surface and pit region (upper arrow). Some cells in a region corresponding to the isthmus also exhibited labelling in the apical cytoplasm (lower arrow). (D) P1: pit cells (red) were located along the luminal surface and in the primordial buds. Small patches of TFF2 expressing epithelial cells (arrow) were found mainly at the luminal surface. (E) P3: TFF2 expressing cells (arrows) were located in the middle of primordial epithelial buds. (F) P60: stomach shows an increase in mucosal thickness and expansion in the area of cells labelled with TFF2 (arrow) which corresponds to the neck region of the gastric glands. (G) P7: pale TFF3 staining (green) was seen in pit cells (red) along the surface and intensified in pit cells forming a mucosal crease (upper left arrow). Deep in the mucosa there were also several scattered parietal cells expressing TFF3 (lower arrows). (H) P21: in addition to pit cells, TFF3 was expressed in zymogenic cells (red) seen at the bottom of the mucosa. The arrow points to a mucosal crease with many Ulex europaeus agglutinin (UEA) labelled (green) pit cells expressing TFF3 (red). Note that away from the mucosal crease, UEA labelled pit cells do not produce TFF3. (I) P60: TFF3 expressing cells (green) were located in the pits forming the mucosal creases (top and lower left) and in zymogenic cells (centre and right side). (J) P3: pit cells (green) were seen along the surface and in the developing epithelial buds. TFF3 staining (red) was seen in pit cells along the surface and in parietal cells (arrows). (K) P21: pit cells (green) were seen along the surface and in the pits of developing nascent glands. TFF3 staining (red) was seen deep in the mucosa where zymogenic cells are located. Bar $=50(A-C), 70(D-K) \mu m$.

(GSII) lectins were used as markers for mucus secreting pit and neck cells, respectively. ${ }^{23}$

\section{Immunogold electron microscopic (EM) analysis}

Small pieces of oxyntic mucosae were fixed in $4 \%$ paraformaldehyde and processed for lowicryl embedding. ${ }^{21}$ Ultrathin sections were mounted on formvar coated nickel grids and probed with polyclonal antibodies specific for TFFs.
Antigen-antibody binding sites were labelled with gold by incubation with goat antirabbit IgG conjugated with 10 or $6 \mathrm{~nm}$ gold particles (Sigma, St Louis, Missouri, USA). Sections were examined by EM.

\section{Protein extraction}

Oxyntic regions and parts of the small intestines were obtained from two pairs of eight month old control and 

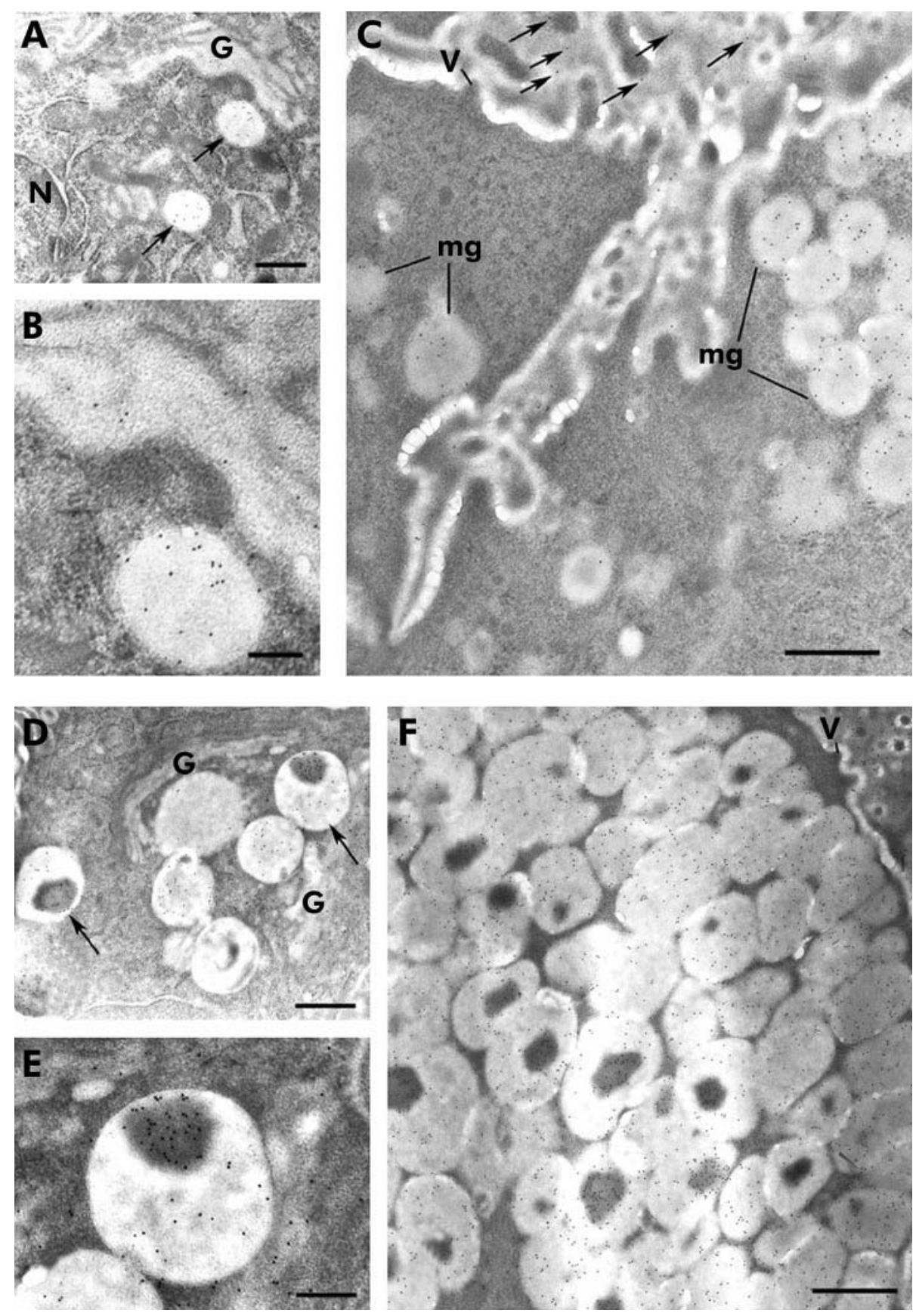

Figure 4 Immunogold localisation of trefoil factor 1 (TFF1) in pre-pit and pit cells of normal adult (P60) oxyntic mucosa. (A) The supranuclear region of a pre-pit cell showing gold particles on the Golgi saccules $(G)$. The contents of two newly produced small secretory granules (arrows) were also covered with gold particles. (B) Close up of the Golgi saccules and upper mucous granule seen in (A). Note the distribution of the black gold particles. (C) Apical cytoplasm of a pre-pit cell (left side) and a differentiating pit cell (right), as seen at the isthmus-pit boundary. The pre-pit cell contained two mucous granules $(\mathrm{mg}$ ) with pale contents labelled with gold particles. The cell projected a few microvilli (v) facing the gland lumen at the top. The differentiating pit cell contained a group of mucous granules $(\mathrm{mg})$. Gold particles cover their pale contents. Note that at the top, some gold particles (arrows) labelled the exocytosed granule contents. (D) Supranuclear region of a pit cell showing gold particles on the Golgi saccules (G) and the newly produced secretory granules. Some of them contained dense core (arrows). (E) Close up of the right cored secretory granule seen in $(A)$ demonstrating the distribution of gold labelled TFF1. Gold particles were numerous in the dark proteinaceous core. (F) Apical cytoplasm of a pit cell facing the gland lumen showing a large group of many secretory granules. Gold particles were scattered on the contents of all granules and were more numerous in the cored granules. The apical membrane of the cell projected a few short microvilli ( $v$ ) into the gland lumen seen at the upper right. Note the presence of gold particles on the exocytosed luminal contents. Bars $=100(B, E), 200(A, D)$, and $300(C, F) \mathrm{nm}$.
TFFl knockout female mice. Tissues were washed in phosphate buffered saline and homogenised in protein extraction buffer $(50 \mathrm{mM}$ Tris $\mathrm{HCl}, \mathrm{pH} 7.4,150 \mathrm{mM} \mathrm{NaCl}$, 1 mM EDTA, 1\% Triton X-100) containing protease inhibitors (Complete; Roche Diagnostics, Mannheim, Germany). After centrifugation for 20 minutes at $10000 \mathrm{~g}$ at $4^{\circ} \mathrm{C}$, supernatants were collected and protein concentrations measured using the Bradford assay (Bio-Rad, Hercules, California, USA). Samples were processed for western blotting and TFF probing, as mentioned above.

\section{RESULTS}

Immunolocalisation of TFFs in the oxyntic mucosa of normal mice

Probing of tissue sections of the developing mouse stomach revealed that TFFI expression occurred as early as postnatal day 1 (P1) when a few labelled cells were found on the surface of the folded epithelial lining (fig 3A). By P3, developing gastric glands appeared and labelled cells increased in number mainly along the luminal surface (fig 3B). When the glands were compartmentalised, at P21, TFFl expressing cells were found along the whole pit region (fig 3C).

To determine the cellular stage at which TFFl expression appears during differentiation of the pit cell lineage, oxyntic mucosal sections of mice at P60 were processed for immunogold labelling and EM examination. TFF 1 was found in pit cell progenitors (pre-pit cells) and subcellular localisation showed that it followed the normal secretory pathway, with gold labelled antigen-antibody binding sites seen within the Golgi saccules, secretory granules, and gland lumen near the apical membrane of pre-pit cells (fig $4 \mathrm{~A}-\mathrm{C}$ ). In mature pit cells, TFFl was also packed in the secretory granules and its 

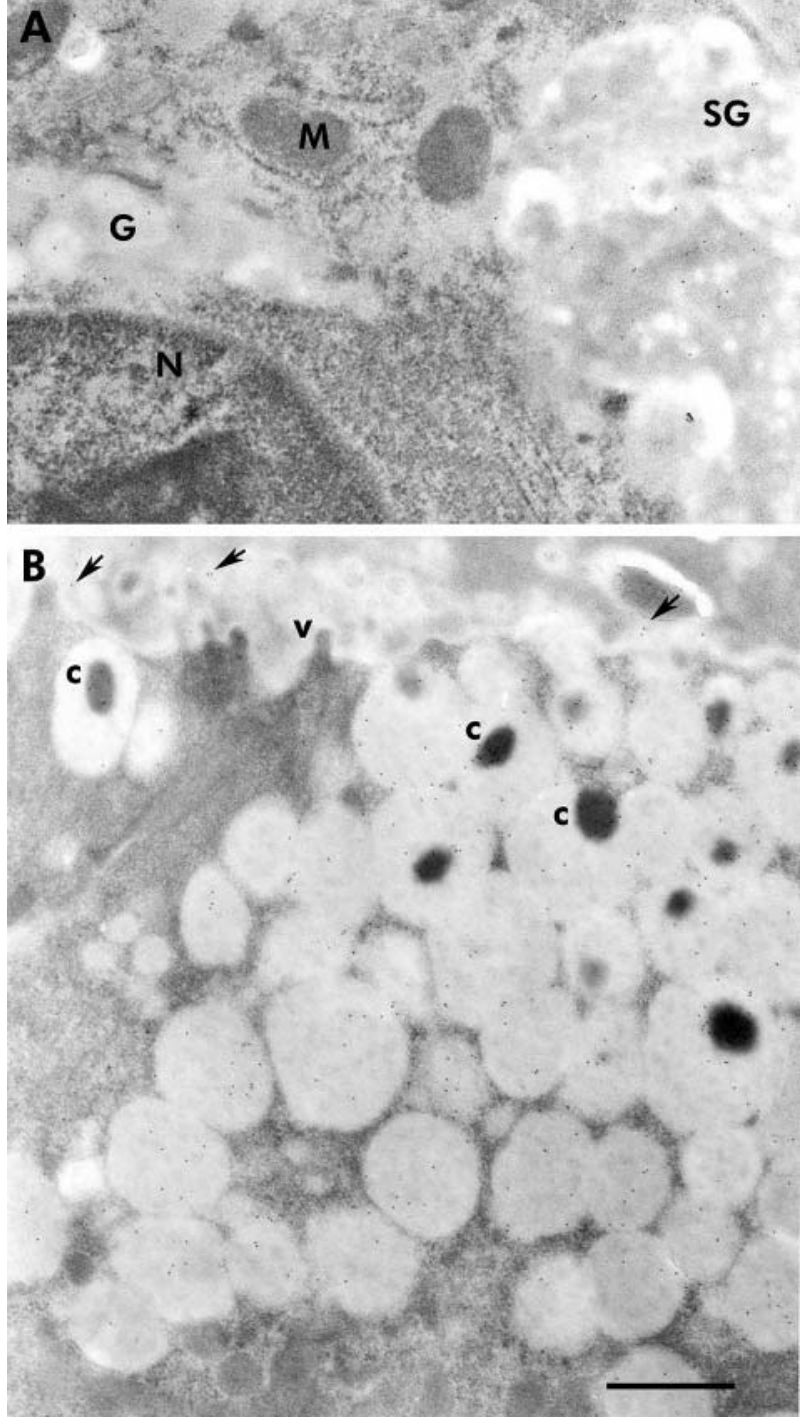

Figure 5 Immunogold localisation of trefoil factors 2 (TFF2) and 3 (TFF3) in normal oxyntic mucosae of adult (postnatal day 60) mice. (A) Supranuclear cytoplasm of a neck cell showing part of the nucleus (N) and the Golgi apparatus (G). Several gold particles were seen on the contents of the Golgi and the newly produced secretory granule (SG), indicating the presence of TFF2. No gold particles were seen on the mitochondria (M). (B) Apical cytoplasm of a pit cell, as seen in the upper segment of the pit. The cytoplasm was packed with many secretory granules and some exhibited a dense core (c). Several gold particles bound to the secretory granule contents. Note that the dense cores exhibited more gold particles than the pale mucinous contents. The apical membrane of the cell projected a few microvilli (v) towards the gland lumen which contained exocytosed secretory material bound to some gold particles (arrows). Bar $=300 \mathrm{~nm}$.

expression became more abundant as cells approached the surface to produce cored granules where TFFl was more abundant in the dark pepsinogenic core than in the pale mucous component (fig 4D-F).

Expression of TFF2 also occurred at P1 in the cytoplasm of a few cells at the luminal surface (fig 3D). By P3, TFF2 was localised in cells within the epithelial folds (fig 3E). In P2l and older mice, TFF2 labelled cells were present in the low isthmus and neck regions (fig $3 \mathrm{~F}$ ). Immunogold localisation of TFF2 confirmed its presence in pre-neck cells and neck cells (fig 5A).

TFF3 expression in developing gastric mucosal tissues appeared only on P7 (fig 3G) in pit and parietal cells. By P21 and in older mice, when the pepsinogen secreting zymogenic cells were developed, labelling was mostly in zymogenic cells and in some pit cells (fig $3 \mathrm{H}, \mathrm{I}$ ). Immunogold localisation of TFF3 confirmed its presence in zymogenic and pit cells (fig 5B). Western blotting of proteins extracted from the stomach corpus and small intestine of adult mice confirmed expression of TFF3 in the stomach (fig 2B) but at a much lower level than in the intestine. To show TFF3 bands, stomach samples had to be loaded 20 -fold more and exposed for approximately 10-fold longer than intestinal samples.

\section{Expression of TFF 2 and TFF 3 in TFF 1 knockout mice}

To test whether expression of TFF2 varies if TFF1 is lacking, oxyntic mucosal sections of TFFl knockout mice were incubated with antibodies specific for TFF2. With the exception of Pl mice, which maintained a low level of expression similar to that of normal $\mathrm{Pl}$ mice, it was not possible to detect any TFF2 in the oxyntic mucosae of all knockout mice examined. This loss of TFF 2 was confirmed by western blotting (fig 2B).

TFF3 expression in TFF1 knockout mice started to appear at P3 $(\mathrm{n}=3)$ in pit and parietal cells (fig $3 \mathrm{~J})$ versus P7 in control mice. In $\mathrm{P} 21$ and in older mice $(\mathrm{n}=3-7$ per time point), TFF3 expressing zymogenic cells were abundant in knockout mice (fig 3K). However, western blotting showed variations in the amounts of protein among samples of control and knockout mice. Some samples showed a decrease in protein level and others an increase (fig 2B, lanes 5 and 6).

\section{Gastric epithelial cell lineage analysis of normal versus TFF 1 deficient mice}

As TFFl is expressed in the pre-pit cell progenitors, its loss in knockout mice could affect the proliferation and commitment programmes of these cells. To test this possibility, sections of oxyntic mucosa of normal and TFFl knockout mice were probed with markers specific for the two cell lineages produced by pre-pit cells: UEA-1 lectin specific for the pit cell lineage and antibodies to the alpha subunit of the proton pump, for the parietal cell lineage.

Normal oxyntic mucosa showed distribution of pit cells along the luminal side of the epithelial unit with parietal cells scattered throughout the unit regions (fig 6A). In knockout mice, there was an expansion in the pit cell population and an apparent reduction in the parietal cell population (fig 6B).

To confirm and provide quantitative data for the change in parietal cell population, cells were counted in at least three different animals per age group. For each animal, counts in 30 longitudinally cut glands were averaged and expressed as cells per gland. Data showed a significant change $(\mathrm{p}<0.01)$ in the number of parietal cells starting from P2l when the glands were compartmentalised. In normal P21 mice, parietal cells averaged nine cells per gland and with TFF 1 loss they decreased to seven cells per gland. The situation was more pronounced in adult mice (P60) when parietal cell number dropped from 15 to nine cells per gland (fig 7).

Labelling of the neck-zymogenic (GSII lectin plus antiintrinsic factor) and enteroendocrine cell lineages in control and knockout mice showed no apparent difference in cellular distribution. Neck and zymogenic cells populated the neck and base regions of the gland whereas enteroendocrine cells were scattered throughout the unit regions (data not shown). Also, mitotic cell labelling showed no apparent difference in control versus knockout mice (fig 1B, C).

\section{DISCUSSION}

The present study demonstrates that, in the oxyntic mucosa of the mouse stomach, (i) TFF1, TFF2, and TFF3 proteins are differentially expressed in a distinct and consistent pattern and (ii) lack of TFF1 affects expression of TFF2 and TFF3, 

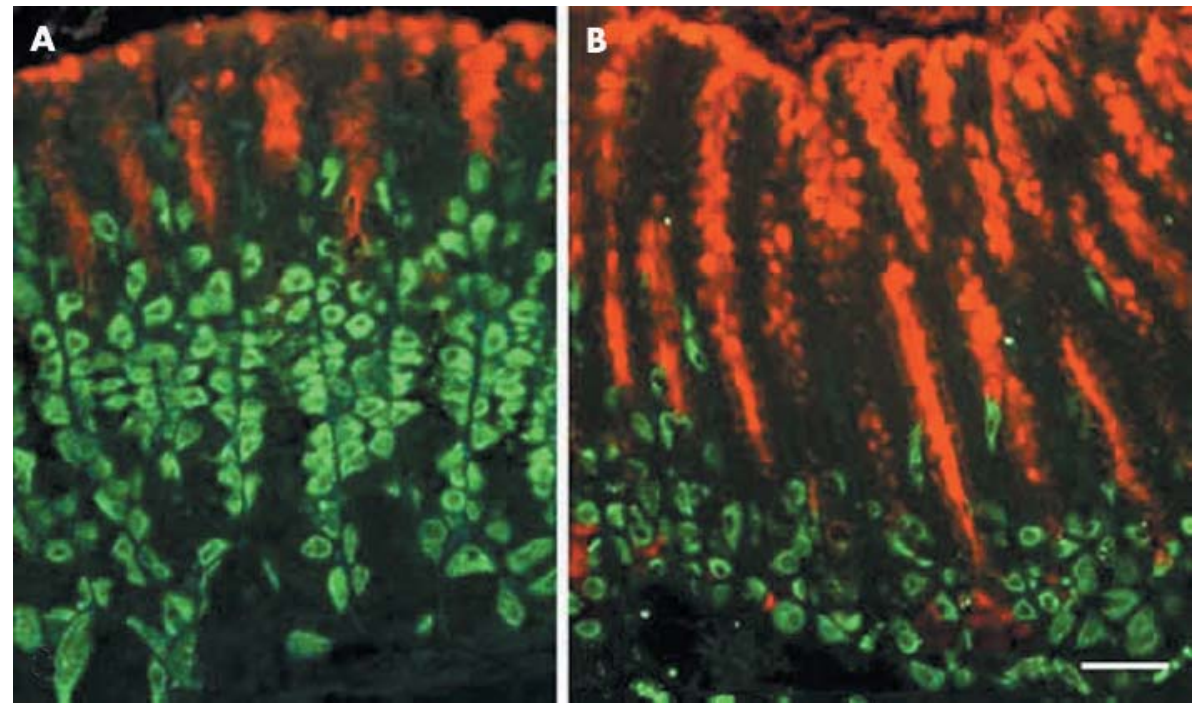

Figure 6 Immunohistological studies of pit and parietal cell lineages in normal and trefoil factor 1 (TFF1) knockout mice. (A) Normal stomach: note that pit cells (red) were seen along the luminal surface and in the pits which form about one third of the mucosal thickness, whereas parietal cells (green) were scattered in the lower two thirds of the mucosa. (b) TFF1 knockout stomach: pit cells expanded and occupied the upper two thirds of the mucosa, whereas parietal cells were reduced in number and became localised mainly in the lower third of the mucosa. Bar $=30 \mu \mathrm{m}$.

and the normal commitment programme of the oxyntic epithelial progenitors.

\section{TFF3 is expressed by the oxyntic mucosa of the mouse stomach}

While expression patterns of TFF1 and TFF2 in postnatal and adult mice are in good agreement with previous data, ${ }^{11}{ }^{12}$ this is not the case for TFF3. In fact, gastric TFF3 mRNA expression has been demonstrated in utero ${ }^{24}$ but most studies report a restricted intestinal expression pattern in the adult gastrointestinal tract (reviewed by Hoffmann and Jagla ${ }^{25}$ ). In the present study, TFF3 appeared at P7 in the developing pit and parietal cells and, at P2 1 when zymogenic cells appeared and the glands became compartmentalised, TFF3 became a feature of zymogenic and pit cells. Finally, in adult mice (P60), using both immunohistochemistry and western blotting, we demonstrated that TFF3 was a product of the mouse oxyntic mucosa. However, the level of TFF3 expression in the oxyntic mucosa was $>100$-fold lower than that in the small intestine. This could explain why TFF3 expression was not previously observed in the stomach, notably using northern blot analysis. ${ }^{7}$

In areas where the oxyntic epithelium deepens into the mucosa and becomes connected to several relatively short

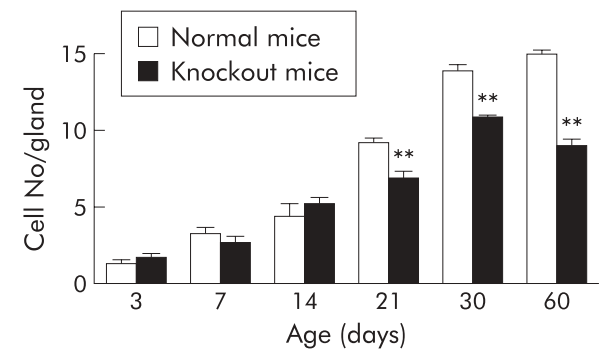

Figure 7 Counts of parietal cells in the oxyntic mucosa of 3-60 day old normal and knockout mice. For each age, three mice were examined, and in each animal, counts from at least 30 longitudinally cut glands were averaged. Data are shown as mean (SEM) cell number per gland. Comparing counts of normal versus knockout mice by the Student's $t$ test showed significant differences $\left({ }^{* *} p<0.01\right)$ at $21-60$ days. glands, pit cells also produce TFF3. This observation may explain how these depressions or creases develop. One possibility is that they are formed when the epithelium is exposed to harsh food particles or any ingested noxious agent, inducing physiological erosion which heals with new cells expressing TFF3. These TFF3 expressing pit cells could be similar to the ulcer associated cell lineage described by Wright and colleagues ${ }^{15}$ in the intestines.

\section{Packaging of TFFs in secretory granules}

Pit cells are produced in the isthmus and migrate towards the luminal surface within two days. During the migration associated differentiation of pit cells, initially their granules are entirely mucous and their turnover time is approximately one hour, but when cells get closer to the surface and reach the upper portion of the pit region, they produce granules which contain a dark core in the mucous background and turnover within four hours. ${ }^{26}$ This core may contain a type of proteinase or lipase, as described in humans ${ }^{27}$ and rabbits, ${ }^{28}$ respectively. The fact that TFF1 and TFF3 are found in the pale mucinous part as well as the dark proteinacious core of the granules indicates that they could play a role in the secretion and/or post-translational modification of these granule components. However, concentrations of both TFFs in the granule core suggests a more significant role related to its contents. In fact, the role of TFFl in protein modification has been recently revealed, as Torres and colleagues ${ }^{29}$ found that TFFl deficiency activates the unfolded protein response. The mucous granules of neck cells are also characterised by a proteinacious core but here it contains pepsinogen. ${ }^{2}$ In this study, the method of fixation used for TFF immunocytochemistry did not preserve this core. Hence we do not know whether there is more TFF2 in the core than in the mucus contents of the granules of neck cells.

\section{Expression of TFF 2 and TFF 3 is affected by TFF 1 deficiency}

As previously reported by Lefebvre and colleagues ${ }^{7}$ in TFF 1 knockout mice, TFF2 expression is abolished as early as P3. In contrast, precocious expression of TFF3 occurs in the parietal cells of these mice ( $\mathrm{P} 3$ versus $\mathrm{P} 7$ in normal mice). By P21, TFF3 is expressed in zymogenic cells of normal and 


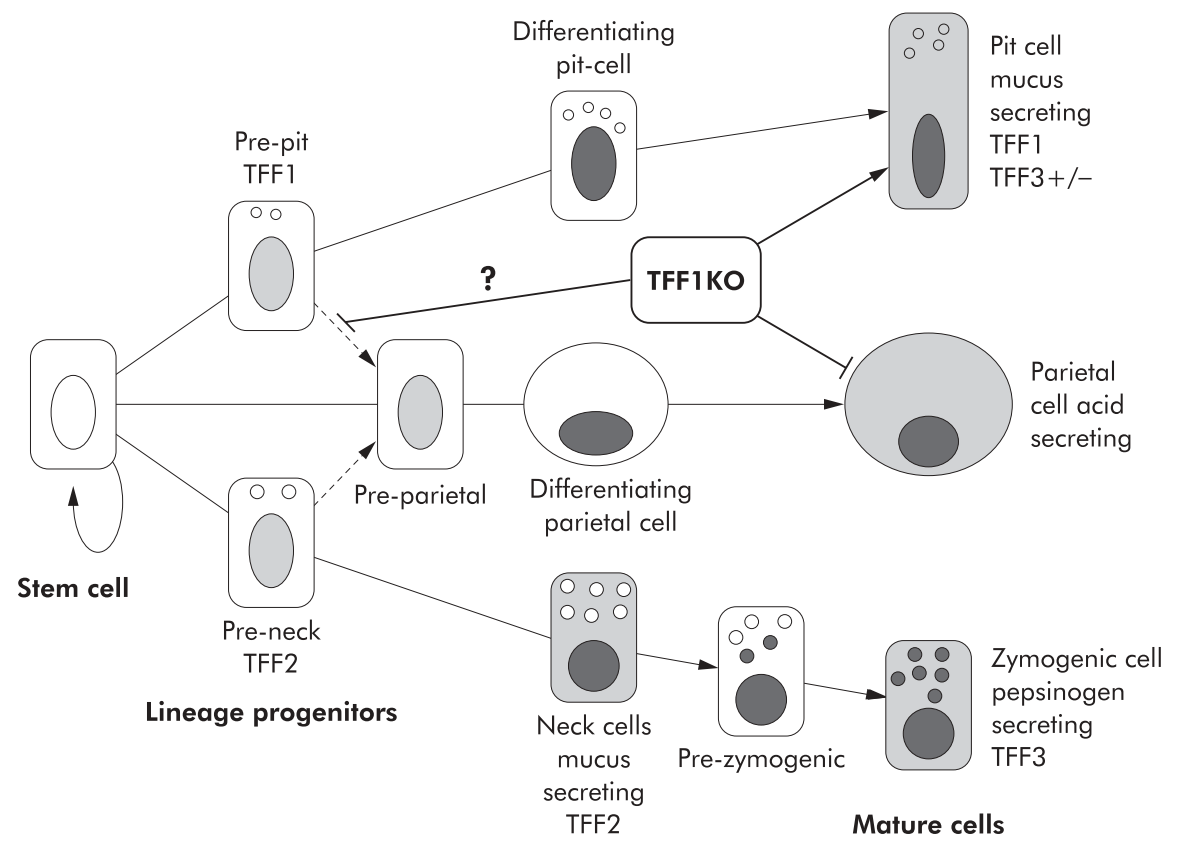

Figure 8 Schematic representation of the effects of trefoil factor 1 (TFF1) knockout (KO) on the commitment programme leading to the three main cell lineages of the oxyntic mucosa. The stem cell normally gives rise to pre-pit, pre-parietal, and pre-neck cells which differentiate into pit, parietal, and neck cells, respectively. The latter changes its phenotype and becomes a zymogenic cell through a pre-zymogenic stage. Note that both pre-pit and preneck cells also give rise to a small proportion of pre-parietal cells. Normal expression of TFF1, TFF2, and TFF3 is indicated. In TFF1 KO animals, the pit cell compartment is increased at the expense of the parietal cell compartment, suggesting that TFF1 is required for pre-pit to pre-parietal commitment.

knockout mice. Western blotting showed inter-animal variability regarding levels of TFF3 in oxyntic mucosa. That lack of expression of one TFF modulates expression of the other two is not surprising as it has been shown that in mice and humans, TFF genes are clustered in a short DNA segment (approximately $40 \mathrm{~kb}$ ) and arranged in the following sequence: TFF1 first then TFF2, followed by TFF3, suggesting that expression of TFF genes may be interrelated. ${ }^{30}{ }^{31}$ In this context, it would be interesting to know whether expression of TFF1 or TFF3 is altered in TFF2 knockout mice. ${ }^{13}$

\section{TFF 1 controls the commitment programme of epithelial} progenitors in the mouse oxyntic mucosa

Lack of TFFl affects the differentiation pathways of lineage progenitors. While neck and zymogenic cells were not altered, both pit and parietal cells were affected. Amplification of the pit cell population occurred early during development and became obvious at $\mathrm{P} 21$, which is the age at which the gastric glands become compartmentalised and isthmus, neck, and base regions become distinctive from the gastric pits. ${ }^{32}$ As pit and parietal cell lineages share a common source of origin (stem cells), amplification of gastric pit cells also occurs if parietal cells are reduced in number ${ }^{33}$ or ablated. ${ }^{34}$ A decrease in the number of parietal cells per gland occurred during development of TFF1 knockout mice and became significant at P21. Parietal cells develop by differentiation of pre-parietal cells which originate from undifferentiated stem cells either directly or via the pre-pit and pre-neck cells. ${ }^{35}$ As TFFl knockout oxyntic mucosa lacks TFF2, we cannot exclude the possibility that the observed phenotype could be TFF2 dependent. However, TFF2 knockout mice do not exhibit the changes described in this study. ${ }^{13}$

\section{Proposed mechanism of TFF 1 action during oxyntic epithelial stem cell commitment}

Factors or molecules involved in the commitment programme of undifferentiated gastric stem cells or pre-pit and preparietal cells have not been discovered to date. Information is starting to evolve with the use of laser capture microdissection and real time quantitative reverse transcription-polymerase chain reaction to identify genes differentially expressed in the progenitor cell zone of mouse gastric glands. Using this powerful technology, Gordon and colleagues have recently released a wealth of information regarding these genes and suggested that insulin-like growth factor is a key player in the growth of these progenitors. ${ }^{36}$ In the same study, they also showed that, in addition to its expression in pit cell region, the TFFl gene is expressed in the progenitor cell zone of mouse oxyntic mucosa. ${ }^{36}$ Here we add that TFFl is a secretory product of pre-pit cells and can only speculate about its role and mechanism of production of more pit cells and fewer parietal cells in TFFl knockout mice. One possibility is that loss of TFFl enhances formation of more pit cells which are the source of some unknown molecules that inhibit production of parietal cells. Another possibility, which is more likely, is that TFFl controls the dual differentiation of pre-pit cells and is necessary for transformation of some prepit cells into parietal cells. Loss of TFFl would lead to transformation of all pre-pit cells into pit cells which, therefore, become numerous and parietal cells would lose one source of origin and decrease in number (fig 8).

While this study confirms expression and cellular localisation of TFF 1 and TFF 2 in pit and neck cells, respectively, it reveals for the first time expression of TFF3 in the mouse oxyntic mucosa. TFFl is a product of pre-pit cells and its deficiency alters their commitment. Due to similarities between the epithelial progenitors of the mouse stomach and those of the rabbit ${ }^{37}$ and human, ${ }^{38}$ it is likely that the role predicted for TFFl is applicable to other species, including humans. In conclusion, TFFl is important for maintaining the normal biology of the oxyntic mucosa and the commitment programme of its epithelial progenitors.

\section{ACKNOWLEDGEMENTS}

We thank Drs MP Chenard and G Buzzell for helpful discussion, and C Wendling, S Tariq, and RR John for technical assistance. This work 
was supported by funds from Terry Fox Foundation (to SMK) and from the Institut National de la Santé et de la Recherche Médicale, the Centre National de la Recherche Scientifique, the Hôpital Universitaire de Strasbourg, the Association pour la Recherche sur le Cancer, and the Ligue Nationale Française contre le Cancer and the Comités du Haut-Rhin et du Bas-Rhin (to MCR and CT).

\section{Authors' affiliations}

S M Karam, Department of Anatomy, Faculty of Medicine and Health Sciences, UAE University, Al-Ain, United Arab Emirates

C Tomasetto, M-C Rio, Institut de Génétique et de Biologie Moléculaire et Cellulaire (IGBMC), CNRS UMR 7104, INSERM U596,ULP, BP 163,

67404 Illkirch Cedex, CU de Strasbourg, France

\section{REFERENCES}

1 Lee ER, Trasler J, Dwivedi S, et al. Division of the mouse gastric mucosa into zymogenic and mucous regions on the basis of gland features. Am J Anat 1982; 164:187-207

2 Karam SM, Leblond CP. Dynamics of epithelial cells in the corpus of the mouse stomach. I. Identification of proliferative cell types and pinpointing of the stem cell. Anat Rec 1993;236:259-79.

3 Del Buono R, Wright NA. The growth of human tumours. In: Peckham M, Pinedo MH, Veronesi U, eds. Oxford textbook of oncology. Oxford: Oxford University Press, 1995:3-12.

4 Wright NA, Hoffmann W, Otto WR, et al. Rolling in the clover: trefoil factor family (TFF)-domain peptides, cell migration and cancer. FEBS Lett 1997;408:121-3.

5 Masiakowski P, Breathnach R, Bloch J, et al. Cloning of cDNA sequences of hormone-regulated genes from the MCF-7 human breast cancer cell line. Nucleic Acids Res 1982;10:7895-903.

6 Rio MC, Bellocq JP, Daniel JY, et al. Breast cancer-associated pS2 protein: synthesis and secretion by normal stomach mucosa. Science 1988;241:705-8

7 Playford RJ, Marchbank T, Goodlad RA, et al. Transgenic mice that overexpress the human trefoil peptide $\mathrm{pS} 2$ have an increased resistance to intestinal damage. Proc Natl Acad Sci U S A 1996;93:2137-42.

8 Lefebvre O, Chenard MP, Masson R, et al. Gastric mucosa abnormalities and tumorigenesis in mice lacking the pS2 trefoil protein. Science 1996;274:259-62

9 Bossenmeyer-Pourie C, Kannan R, Ribieras S, et al. The trefoil factor 1 participates in gastrointestinal cell differentiation by delaying G1-S phase transition and reducing apoptosis. J Cell Biol 2002;157:761-70.

10 Jorgensen KD, Diamant $B$, Jorgensen $\mathrm{KH}$, et al. Pancreatic spasmolytic polypeptide (PSP): III. Pharmacology of a new porcine pancreatic polypeptide with spasmolytic and gastric acid secretion inhibitory effects. Regul Pept 1982;3:231-43.

11 Tomasetto C, Rio MC, Gautier C, et al. hSP, the domain-duplicated homolog of pS2 protein, is co-expressed with pS2 in stomach but not in breast carcinoma. EMBO J 1990;9:407-14

12 Lefebvre $\mathrm{O}$, Wolf $C$, Kedinger $M$, et al. The mouse one P-domain (pS2) and two P-domain (mSP) genes exhibit distinct pattern of expression. J Cell Biol 1993;122:191-8.

13 Farrell JJ, Taupin D, Koh TJ, et al. TFF2/SP-deficient mice show decreased gastric proliferation, increased acid secretion, and increased susceptibility to NSAID injury. J Clin Invest 2002;109:193-204.

14 Rio MC, Chenard MP, Wolf C, et al. Induction of pS2 and hSP genes as markers of mucosal ulceration of the digestive tract. Gastroenterology $1991 ; 100: 375-9$.
15 Wright NA, Pike C, Elia G. Induction of a novel epidermal growth factorsecreting cell lineage by mucosal ulceration in human gastrointestinal stem cells. Nature 1990;343:82-5.

16 Suemori S, Lynch-Devaney K, Podolsky DK. Identification and characterization of rat intestinal trefoil factor: tissue- and cell-specific member of the trefoil protein family. Proc Natl Acad Sci U S A 1991;88:11017-21.

17 Kirikoshi H, Katoh M. Expression of TFF1, TFF2 and TFF3 in gastric cancer. Int J Oncol 2002;21:655-9.

18 Shagger H, von Jagow G. Tricine-sodium dodecyl sulfate-polyacrylamine gel electrophoresis for the separation of proteins in the range from 1 to $100 \mathrm{kDa}$. Anal Biochem 1987; 166:368-79.

19 Smolka A, Swiger KM. Site directed antibodies as topographical probes of the gastric H,K-ATPase alpha-subunit. Biochem Biophys Acta 1992;1 108:75-85.

20 Wen J, Kinnear MB, Richardson MA, et al. Functional expression in Pichia pastoris of human and rat intrinsic factor. Biochim Biophys Acta 2000; 1490:43-53.

21 Tomasetto C, Karam SM, Ribieras S, et al. Identification and characterization of a novel gastric peptide hormone: the motilin-related peptide. Gastroenterology 2000;119:395-405.

22 Cohn SM, Lieberman MW. The use of antibodies to 5-bromo-2'-deoxyuridine for the isolation of DNA sequences containing excision-repair sites. J Biol Chem 1984;259:12456-62.

23 Falk $\mathbf{P}$, Roth KA, Gordon Jl. Lectins are sensitive tools for defining the differentiation programs of mouse gut epitheliual cell lineages. Am J Physiol 1994;266:G987-1003.

24 Otto WR, Patel K. Trefoil factor family (TFF)-domain peptides in the mouse: embryonic gastrointestinal expression and wounding response. Anat Embryol 1999; 199:499-508

25 Hoffmann W, Jagla W. Cell type specific expression of secretory TFF peptides: colocalization with mucins and synthesis in the brain. Int Rev Cytol 2002;213:147-81.

26 Karam SM, Leblond CP. Dynamics of epithelial cells in the corpus of the mouse stomach. II. Outward migration of pit cells. Anat Rec 1993;236:280-96.

27 Samloff IM, Taggart RT, Shiraishi T, et al. Slow moving proteinase. Isolation, characterization, and immunohistochemical localization in gastric mucosa. Gastroenterology 1987:93:77-84.

28 Bernadac A, Moreau H, Verger R. Gastric lipase and pepsinogen during the ontogenesis of rabbit gastric glands. Eur J Cell Biol 1991;55:149-57.

29 Torres LF, Karam SM, Wendling C, et al. Trefoil factor 1 (TFF1/pS2) deficiency activates the unfolded protein response. Mol Med 2002;8:273-82.

30 Ribieras S, Lefebvre O, Tomasetto $C$, et al. Mouse trefoil factor genes: genomic organization, sequences and methylation analyses. Gene $2001 ; 266: 67-75$

31 Gött P, Beck S, Machado JC et al. Human trefoil peptides: genomic structure in 21q22.3 and coordinated expression. Eur J Hum Genet 1996:4:308-15.

32 Karam SM, Li Q, Gordon Jl. Gastric epithelial morphogenesis in normal and transgenic mice. Am J Physiol 1997;272:G1209-20.

33 Konda $Y$, Kamimura $\mathrm{H}$, Yokota $\mathrm{H}$, et al. Gastrin stimulates the growth of gastric pit with less-differentiated features. Am J Physiol 1999;277:G773-84.

34 Li Q, Karam SM, Gordon JI. Diphtheria toxin-mediated ablation of parietal cells in the stomach of transgenic mice. J Biol Chem 1996;271:3671-6.

35 Karam SM. Dynamics of epithelial cells in the corpus of the mouse stomach. IV. Bidirectional migration of parietal cells ending in their gradual degeneration and loss. Anat Rec 1993;236:314-32.

36 Mills JC, Andersson N, Hong CV, et al. Molecular characterization of mouse gastric epithelial progenitor cells. Proc Natl Acad Sci U S A 2002:99:14819-24.

37 Karam SM, Forte JG. Inhibiting gastric $\mathrm{H}(+)-\mathrm{K}(+)$-ATPase activity by omeprazole promotes degeneration and production of parietal cells. Am J Physiol 1994;266:G745-58.

38 Karam SM, Straiton T, Hassan WM, et al. Defining epithelial cell progenitors in the human oxyntic mucosa. Stem Cells 2003;21:322-36.

\section{Making Health Care Safer 2004}

21-22 October 2004

Royal College of Physicians, London

A two day conference for all professionals dedicated to providing safer health care for all. Register now! Early booking discount available.

See website for details: www.quality.bmipg.com 